\title{
Cost-Effectiveness of Maintenance Olaparib for Germline BRCA-Mutated Metastatic Pancreatic Cancer
}

\author{
Bin $\mathrm{Wu}, \mathrm{PhD},{ }^{1}$ and Lizheng Shi, $\mathrm{PhD}^{2}$
}

\begin{abstract}
Background: Maintenance therapy with the PARP inhibitor olaparib for metastatic pancreatic cancer (MPC) with a germline BRCA1 or BRCA2 mutation has been shown to be effective. We aimed to evaluate the cost-effectiveness of maintenance olaparib for MPC from the US payer perspective. Materials and Methods: A partitioned survival model was adopted to project the disease course of MPC. Efficacy and toxicity data were gathered from the Pancreas Cancer Olaparib Ongoing (POLO) trial. Transition probabilities were estimated from the reported survival probabilities in each POLO group. Cost and health preference data were derived from the literature. The incremental cost-utility ratio, incremental net-health benefit, and incremental monetary benefit were measured. Subgroup analysis, one-way analysis, and probabilistic sensitivity analysis were performed to explore the model uncertainties. Results: Maintenance olaparib had an incremental cost-utility ratio of $\$ 191,596$ per additional progression-free survival (PFS) quality-adjusted life-year (QALY) gained, with a high cost of \$132,287 and 0.691 PFS QALY gained, compared with results for a placebo. Subgroup analysis indicated that maintenance olaparib achieved at least a $16.8 \%$ probability of cost-effectiveness at the threshold of $\$ 200,000 /$ QALY. One-way sensitivity analyses revealed that the results were sensitive to the hazard ratio of PFS and the cost of olaparib. When overall survival was considered, maintenance olaparib had an incremental cost-utility ratio of $\$ 265,290$ per additional QALY gained, with a high cost of $\$ 128,266$ and 0.483 QALY gained, compared with results for a placebo. Conclusions: Maintenance olaparib is potentially cost-effective compared with placebo for patients with a germline BRCA mutation and MPC. Economic outcomes could be improved by tailoring treatment based on individual patient factors.
\end{abstract}

J Natl Compr Canc Netw 2020;18(11):1528-1536 doi: $10.6004 /$ jnccn.2020.7587

\footnotetext{
${ }^{1}$ Medical Decision and Economic Group, Department of Pharmacy, Ren Ji Hospital, South Campus, School of Medicine, Shanghai Jiaotong University, Shanghai, China; and ${ }^{2}$ Department of Global Health Management and Policy, School of Public Health and Tropical Medicine, Tulane University, New Orleans, Louisiana.
}

\section{Background}

Pancreatic cancer is the seventh most common cause of cancer death worldwide, accounting for $3.88 \%$ of the disease burden from all neoplasms reported by the Global Burden of Disease Study 2017. ${ }^{1}$ This abysmal statistic is partly attributed to the fact that nearly $50 \%$ of pancreatic cancers are diagnosed as metastatic and approximately $30 \%$ as locoregional disease, with a 5 -year survival rate of $8 \% .^{2}$ For more than a decade, the availability of new drugs and combinations, such as gemcitabinebased chemotherapy, has significantly improved the outcome of patients with metastatic pancreatic cancer (MPC), increasing median overall survival (OS) to 8 to 12 months. ${ }^{3}$ However, therapeutic options for MPC are still limited and the prognosis is poor. ${ }^{4}$

Due to the heterogeneous nature of pancreatic tumors, molecularly targeted therapies could offer physicians the opportunity to tailor a strategy to the unique properties of a patient's individual tumor. ${ }^{2}$ Loss-offunction mutations in BRCA1, BRCA2, or both genes have been found in $4 \%$ to $7 \%$ of patients with pancreatic tumors. ${ }^{5}$ Cells with a BRCA mutation become sensitive to PARP inhibition through multiple mechanisms, including the trapping of PARP on DNA at sites of singlestrand breaks. ${ }^{5}$ As an oral inhibitor of PARP, olaparib can bind the catalytic domain of PARP1, leading to a reduction of PARylation and therefore to a defect in DNA repair. ${ }^{6}$ Olaparib has already been used with success in breast and ovarian cancers. ${ }^{7}$ The Pancreas Cancer Olaparib Ongoing (POLO) trial reported the efficacy and safety of maintenance olaparib compared with placebo in patients with MPC with a germline $B R C A$ mutation. ${ }^{8}$ Results revealed that maintenance olaparib notably prolonged median progression-free survival (PFS) compared with placebo (7.4 vs 3.8 months; hazard ratio [HR] for disease progression or death, 0.53 ; 95\% CI, 0.35-0.82; $P=.004$ ), although no difference in OS between the olaparib and placebo groups was observed. Grade $\geq 3$ adverse drug

See JNCCN.org for supplemental online content. 
events were more frequently reported in the olaparib group than in the placebo group ( $40 \%$ vs $23 \%$ ). Thus, the maintenance olaparib regimen seems to be an attractive option for the treatment of MPC with a germline $B R C A$ mutation. However, considering cost-effectiveness in health decisions is crucial for clinicians and decisionmakers to optimally allocate limited health resources. This analysis aimed to investigate the cost-effectiveness of maintenance olaparib for MPC with a germline $B R C A$ mutation from the US payer perspective.

\section{Materials and Methods}

This study was based on a literature review and modeling techniques and did not require approval by an institutional research ethics board.

\section{Analytic Overview}

The hypothetical target population for this analysis was patients with MPC, a germline BRCA1 or BRCA2 mutation, and disease that had not progressed during first-line platinum-based chemotherapy ( $\geq 16$ weeks of continuous first-line platinum-based chemotherapy), according to the patient characteristics of the POLO trial. ${ }^{8}$ A partitioned-survival model with 3 health states was constructed for an initial decision regarding therapy with maintenance olaparib or placebo. ${ }^{9}$ As shown in Figure 1, the 3 mutually exclusive states were progression-free disease (PFD), progressed disease (PD), and death. In these 3 states, the proportion of OS was partitioned into patients alive and with PFD and patients alive and with PD. The proportion of patients alive at cycle $t$ (a 1-week cycle) was estimated by the area under the OS curve, and the proportion of patients alive and with PFD was estimated by the area under the PFS curve. The proportion of patients alive and with PD was estimated by the difference between the OS and PFS curves. The proportions of PFS and OS were based on the results of the POLO trial, ${ }^{8}$ which was validated by comparing predicted PFS and OS results with the observed data. Patients who initially received olaparib could stop treatment due to either disease progression measure. Because the primary endpoint was PFS and the data maturity of OS was $<50 \%$ in the POLO trial, ${ }^{8}$ the current analysis adopted the cost and health outcomes in the PFS state as the primary endpoints, and the cost and health outcomes in the whole disease course, including PFD, PD, and death, were used as the secondary endpoints.

\section{Clinical Data Inputs}

PFS and OS for maintenance olaparib and placebo were informed by the results of the POLO trial ${ }^{8}$ (ie, the trial follow-up) and extrapolated over the model time horizon using standard statistical analyses described by Guyot et al. ${ }^{10}$ The GetData Graph Digitizer (version 2.26; http:// getdata-graph-digitizer.com) was used to gather the data points from the PFS and OS curves, and these data points were then used to fit the following parametric survival functions: Weibull, log-normal, log-logistic, exponential, generalized gamma, Gompertz and Royston/Parmar spline model, and parametric mixture and nonmixture cure models (see supplemental eTable 1, available with this article at JNCCN.org). The goodness of fit was based on Akaike information criterion. We determined that the Royston/Parmar spline and log-normal model were the most reasonable functions for extrapolating PFS and OS in the placebo arm and that the log-logistic and Royston/Parmar spline models were best for the maintenance olaparib arms. Virtual patient-level data comprised event and censor times and were equal in number to the initial number at risk, which was closely reproduced by digitized Kaplan-Meier curves. The PFS and OS plots created by using virtual patient-level data and the predicted curves created by using parametric survival models are shown in supplemental eFigures 1 and 2 . The influences of the HR of PFS and OS between the maintenance olaparib and placebo groups were checked in sensitivity and subgroup analyses. After the disease progressed, the data of patients who received secondline active treatment were collected from the POLO trial. ${ }^{8}$ The key clinical inputs are summarized in Table 1.
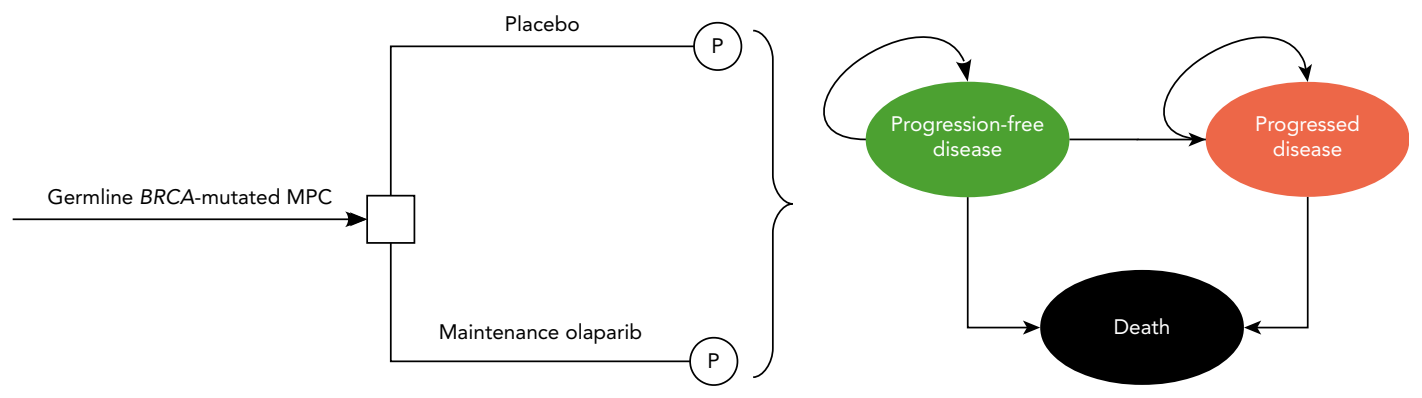

Figure 1. Model structure for germline BRCA-mutated MPC.

Abbreviations: MPC, metastatic pancreatic cancer; P, partitioned survival model. 
Table 1. Model Parameters: Baseline Values, Ranges, and Distributions for Sensitivity Analysis

\begin{tabular}{|c|c|c|c|c|}
\hline Parameter & Expected Value & Range & Distribution & Reference \\
\hline Clinical inputs ${ }^{a}$ & & & & 8 \\
\hline \multicolumn{5}{|l|}{ Survival model of placebo } \\
\hline Royston/Parmar spline model for PFS & \multicolumn{4}{|c|}{ Gamma0, -7.562 (SE, 1.202); gamma1, 2.879 (SE, 0.2915); gamma2, 0.315 (SE, 0.074) } \\
\hline Log-normal model for OS & \multicolumn{4}{|c|}{ Meanlog, 4.355 (SE, 0.131); SDlog, 0.817 (SE, 0.109) } \\
\hline \multicolumn{5}{|l|}{ Survival model of maintenance olaparib } \\
\hline Log-logistic model for PFS & \multicolumn{4}{|c|}{ Shape, 1.9399 (SE, 0.2444); scale, 4.0412 (SE, 0.4932) } \\
\hline Royston/Parmar spline model for OS & \multicolumn{4}{|c|}{ Gamma0, -8.3665 (SE, 1.8360); gamma1, 3.3842 (SE, 0.8936); gamma2, 0.5453 (SE, 0.2351) } \\
\hline HR of PFS between maintenance olaparib and placebo & 0.53 & $0.35-0.82$ & \multicolumn{2}{|l|}{ Log-normal: log-mean, -0.635; log-SD, 2.121} \\
\hline HR of OS between maintenance olaparib and placebo & 0.91 & $0.56-1.46$ & \multicolumn{2}{|l|}{ Log-normal: log-mean, -0.094; log-SD, 1.471} \\
\hline \multicolumn{5}{|l|}{ Proportion of patients receiving subsequent treatment } \\
\hline Placebo & 0.74 & $0.557-0.928$ & \multicolumn{2}{|l|}{ Beta: $\alpha=4.1 ; \beta=1.4$} \\
\hline Maintenance olaparib and placebo & 0.49 & $0.367-0.611$ & \multicolumn{2}{|l|}{ Beta: $\alpha=8.2 ; \beta=8.5$} \\
\hline \multicolumn{5}{|c|}{ Proportion of patients receiving subsequent PARP inhibitor treatment } \\
\hline Placebo & 0.15 & $0.109-0.181$ & \multicolumn{2}{|l|}{ Beta: $\alpha=13.7 ; \beta=80.7$} \\
\hline Maintenance olaparib and placebo & 0.00 & NA & \multicolumn{2}{|l|}{ NA } \\
\hline \multicolumn{5}{|l|}{ Probability of AEs } \\
\hline Any AEs in maintenance olaparib arm & 0.56 & $0.42-0.7$ & \multicolumn{2}{|l|}{ Beta: $\alpha=7 ; \beta=5.5$} \\
\hline Grade $\geq 3$ AEs in maintenance olaparib arm & 0.40 & $0.3-0.5$ & \multicolumn{2}{|l|}{ Beta: $\alpha=9.6 ; \beta=14.4$} \\
\hline Any AEs in placebo arm & 0.70 & $0.525-0.875$ & \multicolumn{2}{|l|}{ Beta: $\alpha=4.8 ; \beta=2.1$} \\
\hline Grade $\geq 3 \mathrm{AEs}$ in placebo arm & 0.23 & $0.173-0.288$ & \multicolumn{2}{|l|}{ Beta: $\alpha=12.3 ; \beta=41.2$} \\
\hline Grade $\geq 3$ fatigue in maintenance olaparib arm & 0.05 & $0.038-0.063$ & \multicolumn{2}{|l|}{ Beta: $\alpha=15.2 ; \beta=288.8$} \\
\hline Grade $\geq 3$ anemia in maintenance olaparib arm & 0.11 & $0.083-0.138$ & \multicolumn{2}{|l|}{ Beta: $\alpha=14.2 ; \beta=115.2$} \\
\hline Grade $\geq 3$ fatigue in placebo arm & 0.02 & $0.015-0.025$ & \multicolumn{2}{|l|}{ Beta: $\alpha=15.7 ; \beta=768.3$} \\
\hline Grade $\geq 3$ anemia in placebo arm & 0.03 & $0.023-0.038$ & \multicolumn{2}{|l|}{ Beta: $\alpha=15.5 ; \beta=501.8$} \\
\hline \multicolumn{5}{|l|}{ Utility inputs } \\
\hline PFD & 0.81 & $0.625-0.995$ & Beta: $\alpha=3.6 ; \beta=0.9$ & 11 \\
\hline PD & 0.58 & $0.396-0.764$ & Beta: $\alpha=4.2 ; \beta=3$ & 12 \\
\hline Disutility due to grade $1-2$ AEs & 0.01 & $0.008-0.02$ & Beta: $\alpha=18 ; \beta=1283.2$ & 13 \\
\hline Disutility due to grade $\geq 3 \mathrm{AEs}$ & 0.16 & $0.11-0.204$ & Beta: $\alpha=36 ; \beta=193$ & 13 \\
\hline \multicolumn{5}{|l|}{ Cost inputs, USD } \\
\hline Olaparib, $600 \mathrm{mg} / \mathrm{d}$ & $\$ 244.52$ & $\$ 122.26-\$ 244.52$ & Fixed & 14 \\
\hline Salvage chemotherapy per month & $\$ 19,379$ & $\$ 17,626-\$ 21,132$ & Gamma: $\alpha=421,281 ; \beta=0.046$ & 15 \\
\hline Supportive care per month & $\$ 1,526$ & $\$ 352-\$ 1,761$ & Gamma: $\alpha=811 ; \beta=1.882$ & 16 \\
\hline Terminal care & $\$ 15,308$ & $\$ 11,481-\$ 19,135$ & Gamma: $\alpha=61,232 ; \beta=0.25$ & 17 \\
\hline Follow-up per month & $\$ 245$ & $\$ 184-\$ 307$ & Gamma: $\alpha=984 ; \beta=0.249$ & 17 \\
\hline Managing anemia per event & $\$ 858$ & $\$ 729-\$ 986$ & Gamma: $\alpha=5,720 ; \beta=0.15$ & $18-21$ \\
\hline Managing fatigue per event & $\$ 750$ & $\$ 563-\$ 938$ & Gamma: $\alpha=2,988 ; \beta=0.251$ & $18-21$ \\
\hline
\end{tabular}

Abbreviations: $A E$, adverse event; $H R$, hazard ratio; OS, overall survival; PD, progressed disease; PFD, progression-free disease; PFS, progression-free survival. ${ }^{a}$ Clinical inputs were all collected from the POLO trial. ${ }^{8}$

\section{Cost and Utility Inputs}

Only direct medical costs were considered and reported in 2018 US dollars, including the drug acquisition costs, costs attributed to the patient's health state, costs for the management of adverse events (AEs), and costs of end-of-life care (Table 1). The costs associated with healthcare services were inflated to 2018 values according to the US Consumer Price Index. ${ }^{11}$

Based on the POLO trial, ${ }^{8}$ olaparib was prescribed at a dose of $300 \mathrm{mg}$ twice daily until disease progression. 
The prices of olaparib in the United States (average wholesale price) were collected from public databases and the literature. ${ }^{12}$ In the United States, the price of olaparib was discounted at $17 \%$ to account for contract pricing. ${ }^{13}$ Because the median relative dose intensity of olaparib was $99.3 \%$ (range of dose intensity, $45 \%-100 \%$ ), ${ }^{8}$ drug wastage cost was not assumed for olaparib in this analysis because whenever a patient with AEs needs to reduce the dose during the treatment cycle, a new drug package must be purchased. After disease progressed, $48.9 \%$ of patients in the olaparib arm and $74.2 \%$ of patients in the placebo arm received subsequent active therapy; $14.5 \%$ of patients in the placebo arm received PARP inhibitor treatment in subsequent therapy. ${ }^{8}$ The cost of salvage chemotherapy was $\$ 19,379$ per patient per month - this cost was derived from a retrospective study including 345 US patients with MPC. ${ }^{14}$ The cost of PARP inhibitor treatment in subsequent therapy was estimated based on the cost of olaparib. The cost of supportive care was $\$ 1,526$ per month. ${ }^{15}$ The costs of follow-up and terminal care were $\$ 245$ and $\$ 15,308$ per month, which were collected from an economic study on advanced pancreatic cancer. ${ }^{16}$

The analysis included costs related to fatigue and anemia-grade 3/4 AEs for which patients in the POLO trial had notably different probabilities. ${ }^{8}$ The unit costs of managing fatigue and anemia were derived from the literature. ${ }^{17-20}$

Each health state was assigned a health utility preference on a scale of 0 (death) to 1 (perfect health). Because the POLO trial ${ }^{8}$ showed that EORTC Core Quality of Life Questionnaire (QLQ-C30) scores were similar between patients receiving maintenance olaparib and those receiving a placebo, we assumed that the health utility preference was only associated with the disease status. The utility preferences of PFD and PD states related to MPC were 0.81 and 0.58 , respectively, which were derived from the EuroQol 5 Dimensions-3 level (EQ-5D-3L) values related to MPC in a Canadian study. ${ }^{21}$ The health utility preference for patients with stable MPC at 8 weeks was adopted for PFD because it was representative of the patients in our analysis. ${ }^{22}$ The disutility values due to grade $1 / 2$ and $3 / 4$ AEs were included in this analysis. ${ }^{23}$ All AEs were assumed to be incurred in the first cycle. The duration-adjusted disutility was subtracted from the baseline PFS utility.

\section{Analysis}

In the base-case analysis, the incremental cost-effectiveness ratio (ICER) and incremental cost-utility ratio (ICUR) were calculated as the incremental cost per additional life-year gained and the additional quality-adjusted lifeyear (QALY) gained for patients taking maintenance olaparib and those taking a placebo, respectively. When the ICUR was lower than the prespecified willingnessto-pay (WTP) threshold ( $\$ 200,000$ per additional QALY gained), cost-effectiveness was assumed according to the recommendation. ${ }^{24}$ Cost and QALYs were discounted at an annual rate of $3 \% .^{25}$ We also estimated the incremental net-health benefit (INHB) and incremental monetary benefit (INMB) based on the following formulas:

$\operatorname{INHB}(\lambda)=\left(\mu_{\mathrm{E} 1}-\mu_{\mathrm{E} 0}\right)-\left(\mu_{\mathrm{C} 1}-\mu_{\mathrm{C} 0}\right) / \lambda=\Delta \mathrm{E}-\Delta \mathrm{C} / \lambda$ and $\operatorname{INMB}(\lambda)=\left(\mu_{\mathrm{E} 1}-\mu_{\mathrm{E} 0}\right) \times \lambda-\left(\mu_{\mathrm{C} 1}-\mu_{\mathrm{C} 0}\right)=\Delta \mathrm{E} \times \lambda-\Delta \mathrm{C}$, where $\mu_{\mathrm{Ci}}$ and $\mu_{\mathrm{Ei}}$ were the cost and effectiveness of maintenance olaparib $(i=1)$ or placebo $(i=0)$, respectively, and $\lambda$ was the WTP threshold $(\$ 200,000 \text { per additional QALY gained })^{26,27}$

Subgroup analysis was performed in the prespecified subgroup as reported in the POLO trial by varying the HRs of PFS. ${ }^{8}$ The model and statistical analyses were implemented in R software (R Foundation for Statistical Computing, version 3.5.2). The data used in this analysis are anonymous and therefore no informed consent was needed.

To evaluate the robustness of the base-case result, 1-way and probabilistic sensitivity analyses were conducted. One-way sensitivity analyses were conducted for all parameters, and the estimated range of each parameter was either based on the reported or estimated $95 \%$ confidence intervals in the referenced studies or determined by assuming a $25 \%$ change from the basecase value (Table 1). In the probabilistic sensitivity analyses, a Monte Carlo simulation of 1,000 iterations was generated by simultaneously sampling the key model parameters from the prespecified distributions. Gamma distribution was selected for the cost parameters, log-normal distribution was used for HRs, and beta distribution was used for probability, proportion, and preference value parameters. Based on the data from the 1,000 iterations, a cost-effectiveness acceptability curve was created to represent the likelihood that maintenance olaparib would be considered cost-effective at various WTP levels for health gains (QALYs).

\section{Results}

\section{Base-Case Analysis and Subgroup Analyses}

In the PFS disease phase, maintenance olaparib provided an additional 0.691 PFS QALYs (approximately 8.3 qualityadjusted life-months) and 0.938 PFS life-years (approximately 11.3 life-months) with an incremental cost of $\$ 132,287$ compared with placebo, which resulted in an ICUR of $\$ 191,596$ per additional PFS QALY gained and an 
ICER of $\$ 141,003$ per additional PFS life-year gained. The INHB in the PFS state was 0.029 QALYs, and the INMB was $\$ 5,803$ at the threshold of $\$ 200,000$ per additional QALY gained (Table 2).

In the whole disease course, maintenance olaparib provided an additional 0.483 QALYs $(\sim 5.8$ quality-adjusted life-months) and 0.579 overall life-years ( $\sim 6.9$ life-months), with an incremental cost of $\$ 128,266$ compared with placebo, which resulted in an ICUR of $\$ 265,290$ per additional QALY gained and an ICER of $\$ 221,789$ per additional life-year gained. The INHB was -0.158 QALYs, and the INMB was $-\$ 31,567$ at the threshold of $\$ 200,000$ per additional QALY gained in the whole disease course (Table 2).

Subgroup analysis by varying the HRs of PFS found that maintenance olaparib presented a positive trend of gaining an INHB in all the subgroups at the threshold of $\$ 200,000$ per additional QALY (Figure 2). The INHBs in the subgroups with respect to the health benefit varied from -0.30 (range, -0.57 to 0.11 ; probability of costeffectiveness, $16.8 \%$ ) in patients aged $\geq 65$ years to 0.24 (range, -0.13 to 0.54 ; probability of cost-effectiveness, 80.3\%) in those with a duration of first-line treatment before randomization of $>6$ months.

\section{Sensitivity Analyses}

The 1-way sensitivity analyses revealed that the HR of PFS played a vital role in model outcomes (Figure 3). When its lower (HR, 0.35) and upper boundaries (HR, 0.82) were adopted, the ICURs of maintenance olaparib versus placebo increased from $\$ 155,913$ per additional
PFS QALY gained to $\$ 353,480$ per additional PFS QALY gained. If the daily cost of olaparib were halved, the ICUR would be less than $\$ 100,000$ per additional PFS QALY. When the utility score of PFD was set at 1 , the ICUR was close to $\$ 150,000$ per additional PFS QALY. Other parameters, such as the cost and utility related to AEs, had only a small impact on the outcome.

The Monte Carlo simulation of 1,000 patients showed that the cost and effectiveness in the PFS disease phase were $\$ 3,124 \pm \$ 1,534$ and $0.905 \pm 0.449$ QALY for placebo and $\$ 139,563 \pm \$ 33,046$ and $1.544 \pm 0.408$ QALY for maintenance olaparib. The cost-effectiveness acceptability curve showed a nearly $54 \%$ probability of maintenance olaparib and $46 \%$ probability of placebo being a cost-effective strategy at the threshold of $\$ 200,000$ per additional QALY gained (Figure 4).

\section{Discussion}

Although oncologists and patients are interested in the clinical benefit from maintenance olaparib treatment noted in the POLO trial due to the increasing incidence of pancreatic cancer, ${ }^{8}$ the high price of an anticancer drug can be a barrier to its access. Health policymakers and payers assess the clinical value of a drug to ensure that patients can access it and that it will be sustainable for national healthcare and reimbursement systems and pharmaceutical companies. ${ }^{28}$ Our study addresses the unmet need for the economic assessment of maintenance olaparib use. ${ }^{29}$ Based on the results of the POLO trial, ${ }^{8}$ our analysis showed that maintenance olaparib

Table 2. Summary of Cost and Outcome Results in Base-Case Analysis

\begin{tabular}{|lcc|} 
& Placebo & Maintenance Olaparib \\
\hline Progression-free cost, USD & $\$ 2,665$ & $\$ 134,952$ \\
\hline Overall cost, USD & $\$ 10,141$ & $\$ 138,407$ \\
\hline Progression-free LYs & 0.950 & 1.888 \\
\hline Overall LYs & 2.094 & 2.673 \\
\hline Progression-free QALYs & 0.726 & 1.417 \\
\hline QALYs & 1.380 & 1.863 \\
\hline ICER (USD per additional PFS LY gained) & & $\$ 141,003$ \\
\hline ICER (USD per additional LY gained)a & & $\$ 221,789$ \\
\hline ICUR (USD per additional PFS QALY gained) & & $\$ 191,596$ \\
\hline ICUR (USD per additional QALY gained) & & $\$ 265,290$ \\
\hline PFS-based INHB (QALY gained) & & \\
\hline PFS-based INMB (USD, money gained) & & 0.029 \\
\hline Overall INHB (QALY gained) & & $\$ 5,803$ \\
\hline Overall INMB (USD, money gained) & & -0.158 \\
\hline
\end{tabular}

Abbreviations: ICER, incremental cost-effectiveness ratio; ICUR, incremental cost-utility ratio; INHB, incremental net-health benefit; INMB, incremental monetary benefit; LY, life-year; PFS, progression-free survival; QALY, quality-adjusted life-year.

${ }^{a}$ Compared with placebo. 


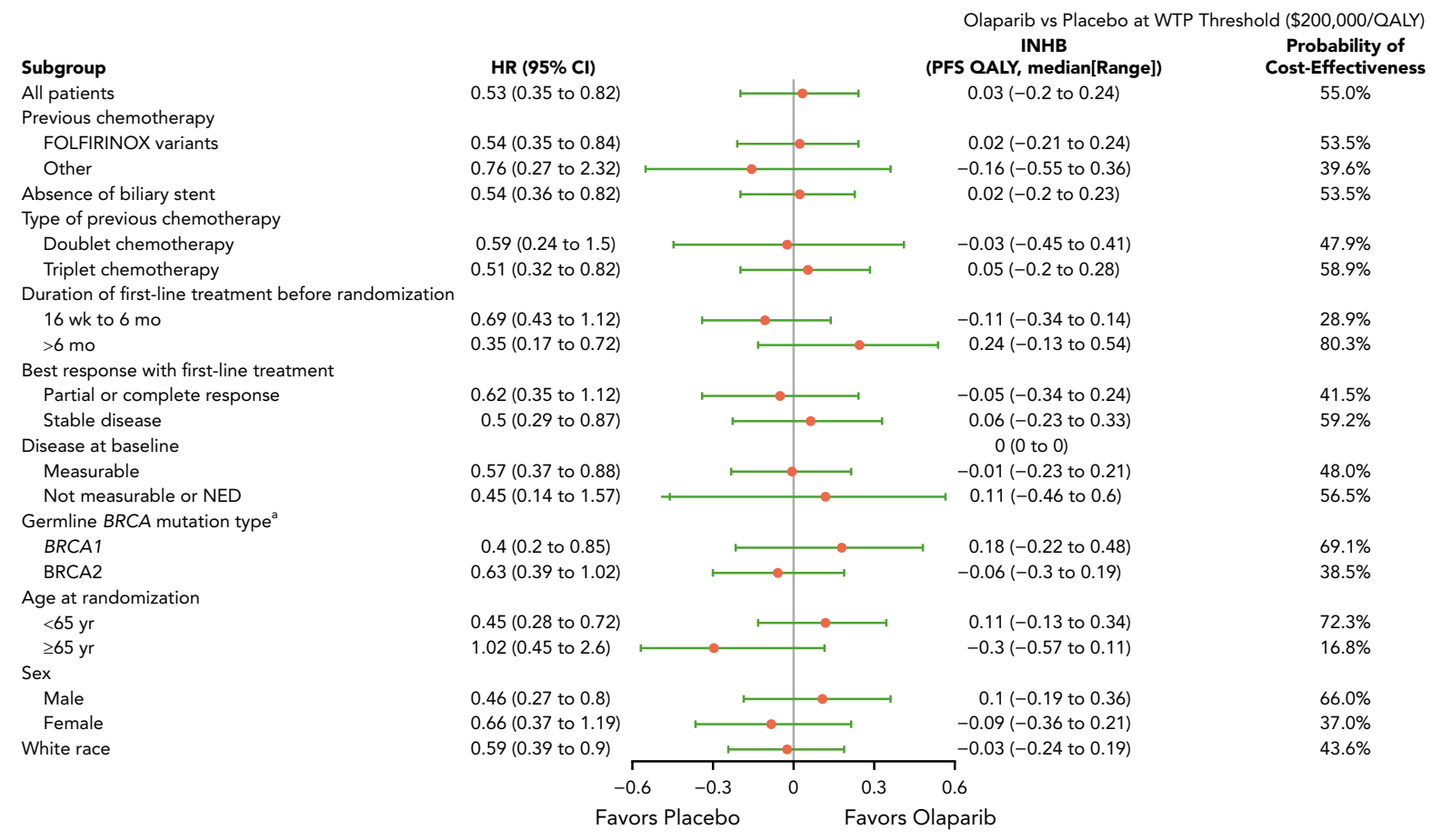

Figure 2. Subgroup analysis of INHB and probabilities of cost-effectiveness by varying HRs of PFS. The vertical line indicates the point of no effect $(\mathrm{INHB}=0)$, the red circle indicates the median INHB, and the green bar indicates the ranges of INHB adjusted by HRs.

Abbreviations: HR, hazard ratio; INHB, incremental net-health benefit; NED, no evidence of disease; PFS, progression-free survival; QALY, quality-adjusted life-year; WTP, willingness to pay.

aDetermined by BRACAnalysis CDx (Myriad Genetics, Inc).

treatment of MPC harboring a $B R C A 1$ or $B R C A 2$ mutation could be optimal for WTP thresholds $<\$ 200,000$ per additional QALY when only the health benefits in the PFS disease phase were considered. Nearly half of the subgroups favored maintenance olaparib treatment because of its positive trend of gaining an INHB compared with placebo.

The nature of olaparib in preventing disease progression was a major driver of economic outcomes. Findings of the one-way sensitivity analysis showed that the HR of PFS is the most sensitive parameter. This result indicated that maintenance olaparib would become more cost-effective in patients with a more favorable HR of PFS, such as those harboring a BRCAl mutation and receiving $>6$ months of chemotherapy before initiating olaparib treatment. However, in some patients with a more unfavorable HR of PFS with a high risk of progression, such as those aged $\geq 65$ years, maintenance olaparib may be less cost-effective.

Because there was no statistical difference in OS between patients treated with olaparib and versus a placebo, maintenance olaparib treatment during the whole disease course was not cost-effective because its ICUR was higher than the threshold of $\$ 200,000$ per additional QALY. This lack of cost-effectiveness can be partly explained by the fact that longer duration of olaparib treatment and higher cost associated with the PD state in the olaparib arm could more substantially compensate for the high cost of olaparib treatment in the PFS state compared with the placebo arm. However, the compensation was not substantial in the current analysis because of the short duration of the PD state in the olaparib arm. The cost of olaparib was also found to be an important influential factor. When the daily cost of olaparib decreased by $50 \%$, the ICUR for maintenance olaparib decreased to a level lower than $\$ 200,000 /$ QALY. Recently, the US government has proposed indexing the prices that Medicare pays for drugs to those paid by health systems in other developed countries to help reduce the relatively high prices paid by US patients. ${ }^{30}$ Once this proposal is enacted or implemented, the initiative may lead to a reduction in the price of olaparib and to achieving more favorable economic outcomes.

The strengths of this study are worth highlighting. To our knowledge, this is the first analysis to simultaneously evaluate the economic outcomes of olaparib for the maintenance treatment of MPC harboring a germline $B R C A$ mutation by synthesizing the latest evidence through an economic modeling approach. Maintenance treatment is a new concept in MPC, although maintenance 


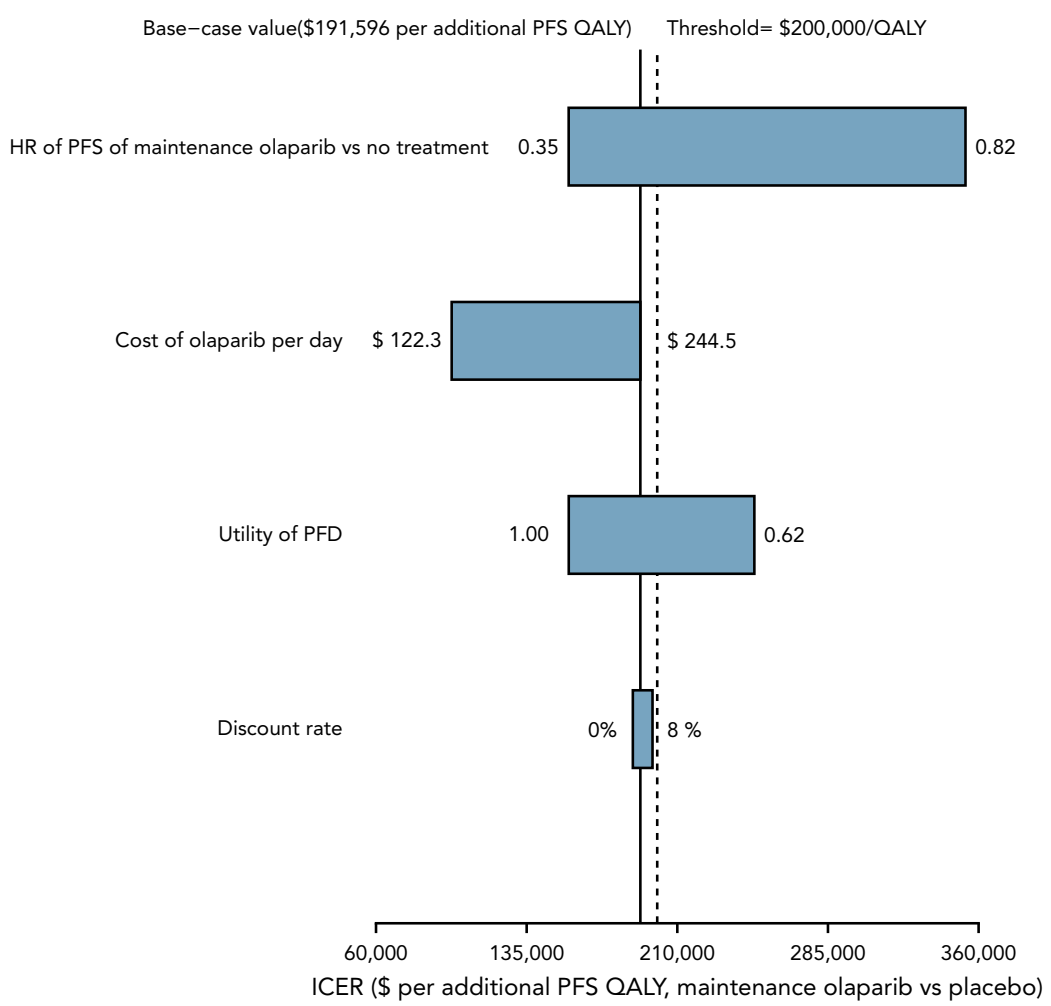

Figure 3. Tornado diagram of one-way sensitivity analyses of maintenance olaparib versus placebo.

Abbreviations: HR, hazard ratio; ICER, incremental cost-effectiveness ratio; PFD, progression-free disease; PFS, progression-free survival; QALY, quality-adjusted life-year.

capecitabine and fluorouracil have shown promising results in early trials. ${ }^{31,32}$ However, there is a dearth of information regarding the economic outcome of maintenance treatments for MPC. Although 2 previous economic analyses have shown that a chemotherapy regimen containing nab-paclitaxel was cost-effective in the United States and United Kingdom, ${ }^{20,33,34}$ these analyses included treatment-naïve patients with MPC. The target populations in those studies were different from ours in that the patients we studied had received at least 16 weeks of continuous first-line platinum-based chemotherapy. Second, the current analysis examined the economic outcomes of 19 subgroups prespecified by the POLO trial. ${ }^{8}$ The information about subgroup economic analysis would be helpful for physicians and patients.

Several weaknesses in the analysis should be noted. First, because of the lack of data, we did not include other PARP inhibitors and chemotherapeutic agents as maintenance treatment. New PARP inhibitors such as niraparib have shown favorable economic outcomes as maintenance treatment in other cancers with germline $B R C A$ mutations. ${ }^{35}$ The current analysis needs to be updated when evidence is available. Second, we explored the health benefits beyond the observation time of the POLO trial through the fitting of parametric distributions to the reported survival data. Because the data maturity of OS was lower than $46 \%$, the current analysis needs to be updated when the data become mature. Third, we did

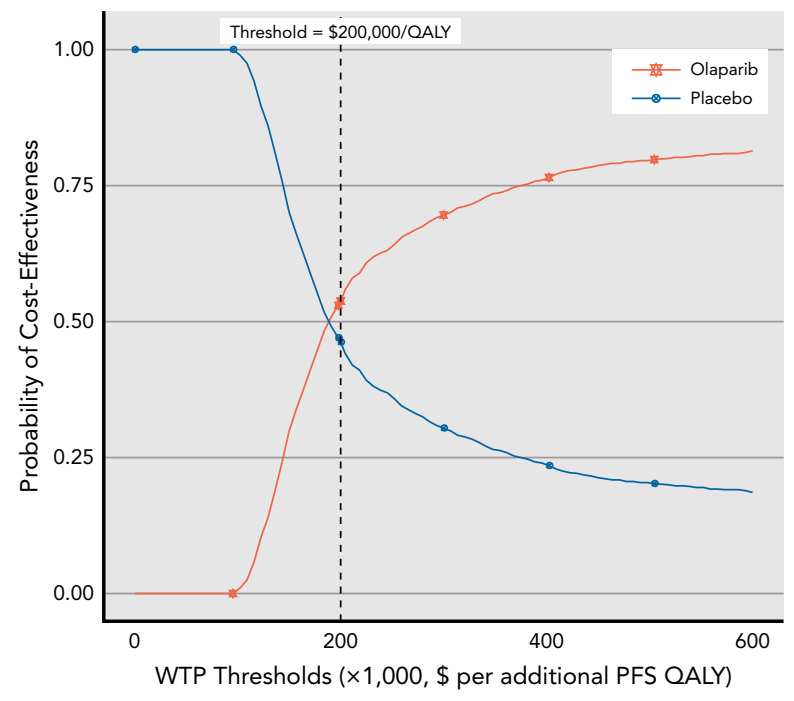

Figure 4. Cost-effectiveness acceptability curves of maintenance olaparib versus placebo.

Abbreviations: PFS, progression-free survival; QALY, quality-adjusted life-year; WTP, willingness to pay. 
not measure the budget impact of maintenance olaparib on society. A long-lasting prescription of olaparib may raise the financial burden extremely high. Because approximately one-half of the 56,770 patients newly diagnosed with pancreatic cancer each year will be diagnosed with advanced disease with a $7.5 \%$ prevalence of a germline $B R C A$ mutation, ${ }^{8,36}$ we estimated that nearly 1,700 patients would be eligible for maintenance olaparib treatment after 4 months of first-line chemotherapy. Fourth, the current analysis adopted a prespecified WTP threshold of $\$ 200,000$ per additional QALY gained because an ICER of $\$ 200,000$ per additional QALY gained, or $<3$ times the annual gross domestic product per capita, are commonly used thresholds to determine the cost-effectiveness of cancer treatment. However, note that there is no official WTP threshold recognized in the United States. When a threshold of $<\$ 100,000$ per additional QALY gained is adopted, maintenance olaparib will become not cost-effective. Thus, the final conclusions should be carefully explained and referenced. Finally, the costs of grade $1 / 2$ AEs were excluded from the evaluation, which may overestimate the economic results of maintenance olaparib. This weakness may not be a major one, as implied by the findings in the 1-way sensitivity analysis, which indicated that the costs related to AEs had only a small impact. However, because the results of this evaluation reflect the general clinical conditions of managing MPC, this finding may be a valuable reference for physicians and policymakers.

\section{Conclusions}

Our estimates show that maintenance olaparib is potentially a cost-effective option for patients with MPC harboring a germline $B R C A$ mutation from a US payer perspective. Economic outcomes could be improved by tailoring treatment based on individual patient factors. These findings may help clinicians make optimal treatment decisions for patients with MPC. Because of the methodological flaws in the present study, more quality clinical and economic real-world data are needed in this area; we believe that this focus will provide more sound evidence as a framework for determining the value of different therapeutic alternatives in oncology.

\section{Submitted August 22, 2019; accepted for publication May 8, 2020}

Author contributions: Study design, data collection, economic analysis: All authors. Manuscript preparation: Wu. Critical revision: Shi.

Disclosures: The authors have disclosed that they have not received any financial consideration from any person or organization to support the preparation, analysis, results, or discussion of this article.

Correspondence: Lizheng Shi, PhD, Department of Global Health Management and Policy, School of Public Health and Tropical Medicine, Tulane University, 1440 Canal Street, Suite 1900, New Orleans, LA 70112. Email: Ishi1@tulane.edu

\section{References}

1. GBD 2017 DALYs and HALE Collaborators. Global, regional, and national disability-adjusted life-years (DALYs) for 359 diseases and injuries and healthy life expectancy (HALE) for 195 countries and territories, 19902017: a systematic analysis for the Global Burden of Disease Study 2017. Lancet 2018;392:1859-1922.

2. Sheahan $A V$, Biankin $A V$, Parish $C R$, et al. Targeted therapies in the management of locally advanced and metastatic pancreatic cancer: a systematic review. Oncotarget 2018;9:21613-21627.

3. Chan K, Shah K, Lien K, et al. A Bayesian meta-analysis of multiple treatment comparisons of systemic regimens for advanced pancreatic cancer. PLoS One 2014;9:e108749.

4. Kamisawa T, Wood LD, Itoi T, et al. Pancreatic cancer. Lancet 2016;388: 73-85.

5. O'Sullivan CC, Moon DH, Kohn EC, et al. Beyond breast and ovarian cancers: PARP inhibitors for BRCA mutation-associated and BRCA-like solid tumors. Front Oncol 2014;4:42.

6. Lodovichi S, Mercatanti A, Cervelli T, et al. Computational analysis of data from a genome-wide screening identifies new PARP1 functional interactors as potential therapeutic targets. Oncotarget 2019;10: 2722-2737.

7. Bao Z, Cao C, Geng X, et al. Effectiveness and safety of poly (ADP-ribose) polymerase inhibitors in cancer therapy: a systematic review and metaanalysis. Oncotarget 2016;7:7629-7639.

8. Golan T, Hammel P, Reni M, et al. Maintenance olaparib for germline BRCA-mutated metastatic pancreatic cancer. N Engl J Med 2019;381: 317-327.

9. Williams C, Lewsey JD, Mackay DF, et al. Estimation of survival probabilities for use in cost-effectiveness analyses: a comparison of a multi-state modeling survival analysis approach with partitioned survival and Markov decision-analytic modeling. Med Decis Making 2017;37:427-439

10. Guyot P, Ades AE, Ouwens MJ, et al. Enhanced secondary analysis of survival data: reconstructing the data from published Kaplan-Meier survival curves. BMC Med Res Methodol 2012;12:9.
11. U.S. Bureau of Labor Statistics. CPI Inflation Calculator. Accessed January 5, 2019. Available at: https://www.bls.gov/data/inflation_calculator.htm

12. IBM Corporation. IBM Micromedex web applications access. Accessed May 13, 2020. Available at: http://www.micromedexsolutions.com

13. Hornberger J, Hirsch FR, Li Q, et al. Outcome and economic implications of proteomic test-guided second- or third-line treatment for advanced non-small cell lung cancer: extended analysis of the PROSE trial. Lung Cancer 2015;88:223-230.

14. Kim GP, Parisi MF, Patel MB, et al. Comparison of treatment patterns, resource utilization, and cost of care in patients with metastatic pancreatic cancer treated with first-line nab-paclitaxel plus gemcitabine or FOLFIRINOX. Expert Rev Clin Pharmacol 2017;10:559-565.

15. O'Neill CB, Atoria $C L, O^{\prime}$ Reilly EM, et al. Costs and trends in pancreatic cancer treatment. Cancer 2012;118:5132-5139.

16. Murphy JD, Chang DT, Abelson J, et al. Cost-effectiveness of modern radiotherapy techniques in locally advanced pancreatic cancer. Cancer 2012;118:1119-1129.

17. Perrin A, Sherman S, Pal S, et al. Lifetime cost of everolimus vs axitinib in patients with advanced renal cell carcinoma who failed prior sunitinib therapy in the US. J Med Econ 2015;18:200-209.

18. Hansen RN, Hackshaw MD, Nagar SP, et al. Health care costs among renal cancer patients using pazopanib and sunitinib. J Manag Care Spec Pharm 2015;21:37-44.

19. Liou SY, Stephens JM, Carpiuc KT, et al. Economic burden of haematological adverse effects in cancer patients: a systematic review. Clin Drug Investig 2007;27:381-396.

20. Gharaibeh M, McBride A, Bootman JL, et al. Economic evaluation for the US of nab-paclitaxel plus gemcitabine versus FOLFIRINOX versus gemcitabine in the treatment of metastatic pancreas cancer. J Med Econ 2017; 20:345-352.

21. Lien K, Tam VC, Ko YJ, et al. Impact of country-specific EQ-5D-3L tariffs on the economic value of systemic therapies used in the treatment of metastatic pancreatic cancer. Curr Oncol 2015;22:e443-452. 
22. Attard CL, Brown S, Alloul K, et al. Cost-effectiveness of FOLFIRINOX for first-line treatment of metastatic pancreatic cancer. Curr Oncol 2014;21: e41-51.

23. Amdahl J, Diaz J, Park J, et al. Cost-effectiveness of pazopanib compared with sunitinib in metastatic renal cell carcinoma in Canada. Curr Oncol 2016;23:e340-354.

24. Neumann PJ, Cohen JT, Weinstein MC. Updating cost-effectiveness-the curious resilience of the $\$ 50,000$-per-QALY threshold. N Engl J Med 2014; 371:796-797.

25. Sanders GD, Neumann PJ, Basu A, et al. Recommendations for conduct, methodological practices, and reporting of cost-effectiveness analyses: second panel on cost-effectiveness in health and medicine. JAMA 2016; 316:1093-1103.

26. Craig BA, Black MA. Incremental cost-effectiveness ratio and incremental net-health benefit: two sides of the same coin. Expert Rev Pharmacoecon Outcomes Res 2001;1:37-46.

27. Stinnett AA, Mullahy J. Net health benefits: a new framework for the analysis of uncertainty in cost-effectiveness analysis. Med Decis Making 1998;18(2 Suppl):S68-80.

28. Uyl-de Groot CA, Löwenberg B. Sustainability and affordability of cancer drugs: a novel pricing model. Nat Rev Clin Oncol 2018;15:405-406.
29. Gérard C, Fagnoni $P$, Vienot $A$, et al. A systematic review of economic evaluation in pancreatic ductal adenocarcinoma. Eur J Cancer 2017;86:207-216.

30. Dyer O. US drug prices should be tied to foreign prices to tackle "global freeloading," says Trump. BMJ 2018;363:k4542.

31. Hann A, Bohle W, Egger J, et al. Feasibility of alternating induction and maintenance chemotherapy in pancreatic cancer. Sci Rep 2017;7:41549

32. Reure J, Follana P, Gal J, et al. Effectiveness and tolerability of maintenance capecitabine administrated to patients with metastatic pancreatic cancer treated with first-line FOLFIRINOX. Oncology 2016;90:261-266.

33. Gharaibeh M, McBride A, Alberts DS, et al. Economic evaluation for USA of systemic chemotherapies as first-line treatment of metastatic pancreatic cancer. Pharmacoeconomics 2018;36:1273-1284.

34. Gharaibeh M, McBride A, Alberts DS, et al. Economic evaluation for the UK of systemic chemotherapies as first-line treatment of metastatic pancreatic cancer. Pharmacoeconomics 2018;36:1333-1343.

35. Guy H, Walder L, Fisher M. Cost-effectiveness of niraparib versus routine surveillance, olaparib and rucaparib for the maintenance treatment of patients with ovarian cancer in the United States. Pharmacoeconomics 2019;37:391-405

36. Siegel RL, Miller KD, Jemal A. Cancer statistics, 2019. CA Cancer J Clin 2019;69:7-34

\section{See JNCCN.org for supplemental online content.}

\section{SUBSCRIBE TO JNCCN TODAY!}

To receive your complimentary print subscription of JNCCN-Journal of the National Comprehensive Cancer Network, go to www.jncen.org/page/subscribe or call the Circulation Department at (631) 935-7651 to see if you qualify. 
Supplemental online content for:

\section{Cost-Effectiveness of Maintenance Olaparib for Germline BRCA-Mutated Metastatic Pancreatic Cancer}

Bin $\mathrm{Wu}, \mathrm{PhD}$, and Lizheng Shi, PhD

J Natl Compr Canc Netw 2020;18(11):1528-1536

eFigure 1: Replicated Kaplan-Meier PFS Curves of Maintenance Olaparib and Placebo in the POLO Trial eFigure 2: Replicated Kaplan-Meier OS Curves of Maintenance Olaparib and Placebo in the POLO Trial eFigure 3: Probabilistic Results of the Incremental Cost-Effectiveness Difference for Maintenance Olaparib Versus Placebo

eTable 1: Estimated Parameters and Akaike Information Criterion Values From Each Survival Model 


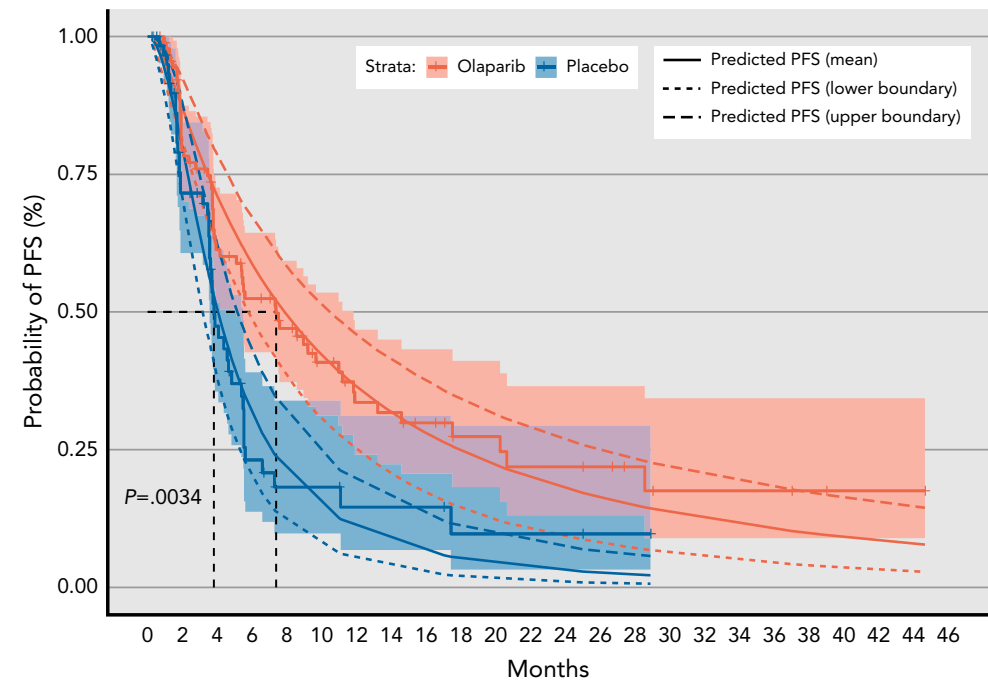

Number at risk

$\begin{array}{lllllllllllllllllllllllllll}\text { Olaparib } & 92 & 69 & 50 & 41 & 34 & 24 & 18 & 17 & 14 & 10 & 10 & 8 & 8 & 7 & 5 & 3 & 3 & 3 & 3 & 2 & 1 & 1 & 1 & 0\end{array}$

Placebo $\quad \begin{array}{lllllllllllllllllllllllll} & 39 & 23 & 10 & 6 & 6 & 4 & 4 & 4 & 2 & 2 & 2 & 2 & 1 & 1 & 0 & 0 & 0 & 0 & 0 & 0 & 0 & 0 & 0\end{array}$

eFigure 1. Replicated Kaplan-Meier PFS curves of maintenance olaparib and placebo in the POLO trial, based on distribution information shown in Table 1. The solid smooth line indicates the predicted PFS (mean), and the dotted and dashed lines indicate the $95 \%$ upper and lower predicted PFS.

Abbreviation: PFS, progression-free survival.

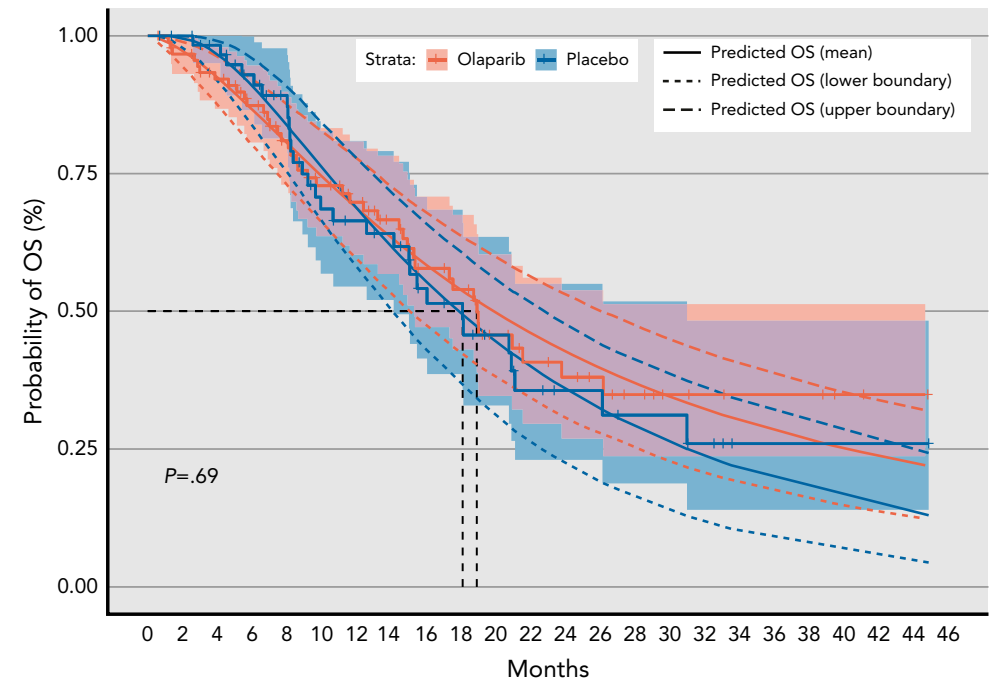

Number at risk

$\begin{array}{lllllllllllllllllllllllll}\text { Olaparib } & 92 & 87 & 80 & 71 & 61 & 51 & 46 & 39 & 31 & 28 & 20 & 16 & 14 & 12 & 9 & 6 & 5 & 4 & 4 & 4 & 2 & 1 & 1 & 0\end{array}$

$\begin{array}{llllllllllllllllllllllllllllllllll}\text { Placebo } & 62 & 60 & 56 & 50 & 44 & 32 & 29 & 27 & 20 & 18 & 14 & 10 & 8 & 8 & 6 & 6 & 4 & 1 & 1 & 1 & 1 & 1 & 1 & 0\end{array}$

eFigure 2. Replicated Kaplan-Meier OS curves of maintenance olaparib and placebo in the POLO trial, based on distribution information shown in Table 1. The solid smooth line indicates the predicted OS (mean), and the dotted and dashed lines indicate the $95 \%$ upper and lower predicted OS.

Abbreviation: OS, overall survival. 


\section{2 - Wu and Shi}

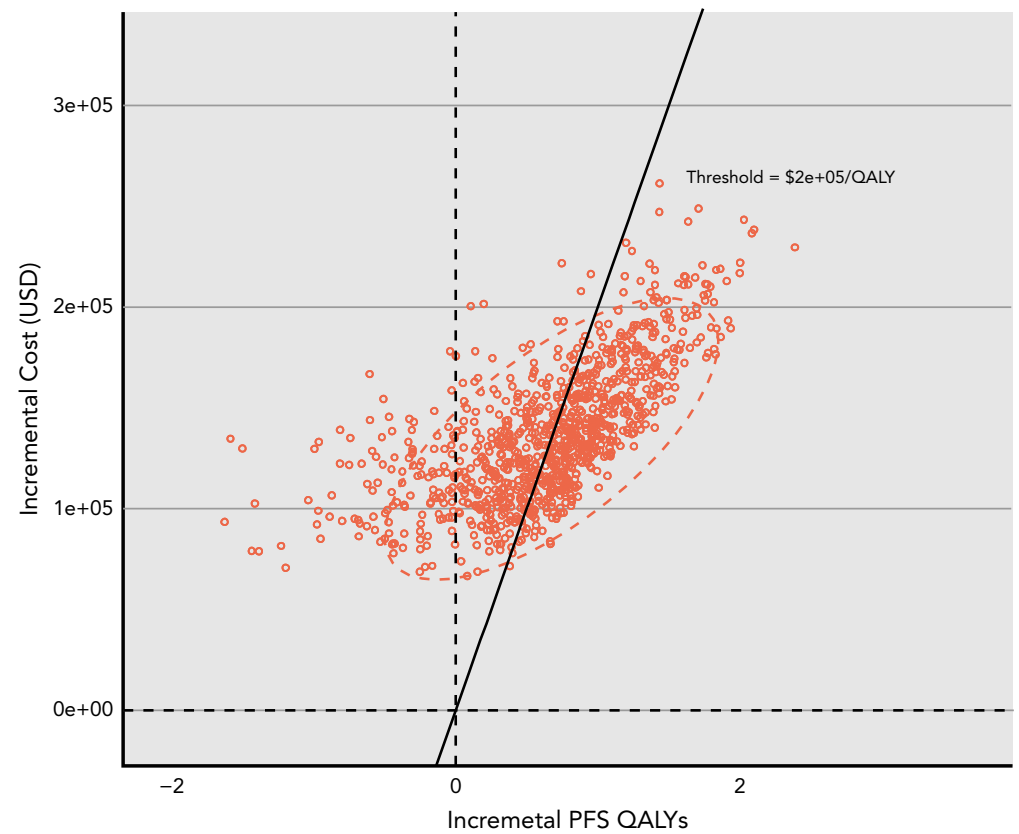

eFigure 3. Probabilistic results of the incremental cost-effectiveness difference for maintenance olaparib versus placebo. The y axis represents the incremental costs, whereas the $x$ axis represents the incremental QALYs gained. The red ellipses surround $95 \%$ of the estimates.

Abbreviations: PFS, progression-free survival; QALY, quality-adjusted life-year. 
eTable 1. Estimated Parameters and AIC Values From Each Survival Model

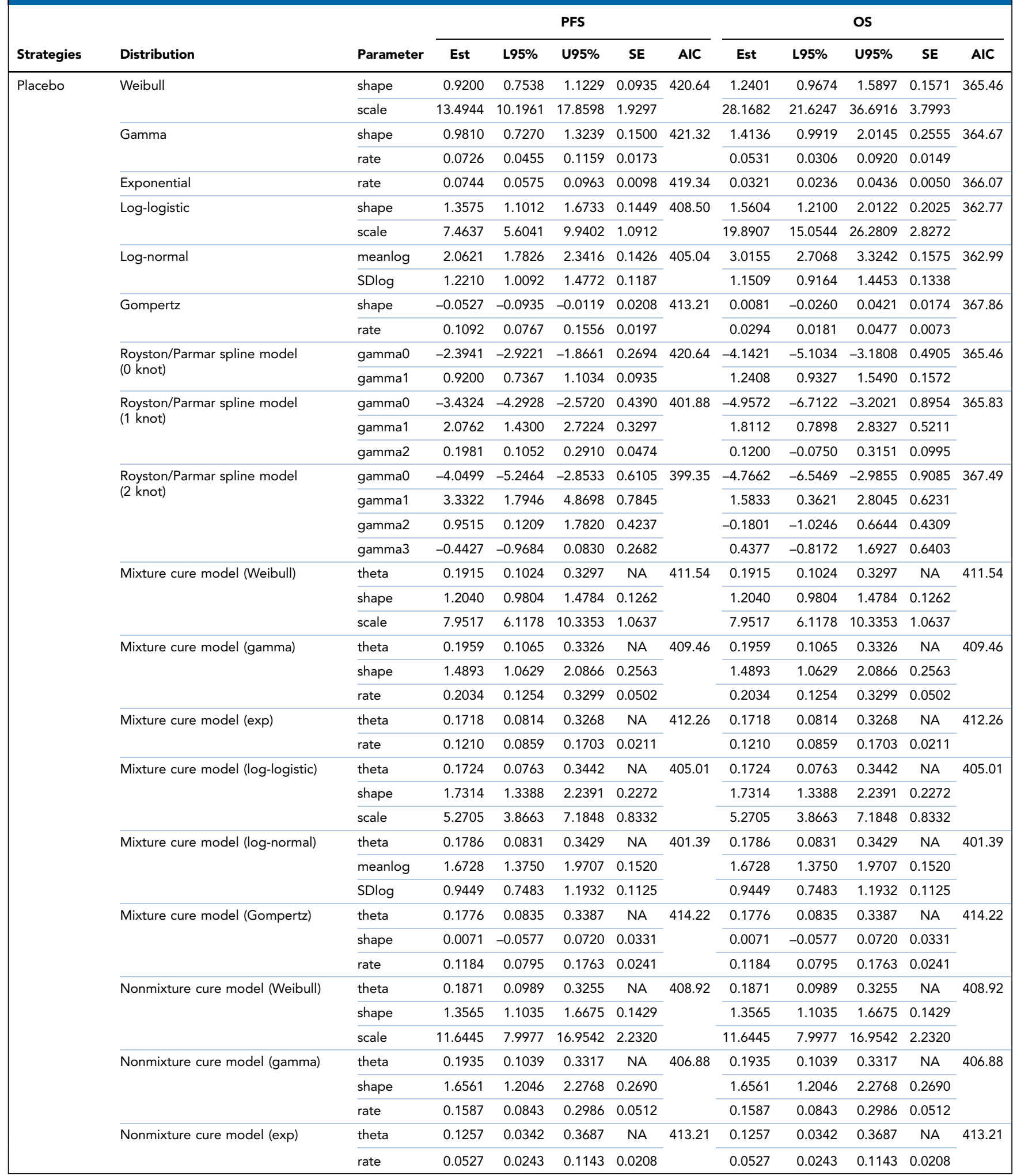


eTable 1. Estimated Parameters and AIC Values From Each Survival Model (cont.)

PFS

os

\begin{tabular}{|c|c|c|c|c|c|c|c|c|c|c|c|c|}
\hline \multirow[b]{2}{*}{ Strategies } & \multirow[b]{2}{*}{ Distribution } & \multirow[b]{2}{*}{ Parameter } & & \\
\hline & & & Est & L95\% & U95\% & SE & AIC & Est & L95\% & U95\% & SE & AIC \\
\hline \multirow[t]{9}{*}{ Placebo (cont.) } & \multirow[t]{3}{*}{ Nonmixture cure model (log-logistic) } & theta & 0.1813 & 0.0860 & 0.3425 & NA & \multirow[t]{3}{*}{405.70} & 0.1813 & 0.0860 & 0.3425 & NA & \multirow[t]{3}{*}{405.70} \\
\hline & & shape & 1.6707 & 1.2793 & 2.1819 & 0.2276 & & 1.6707 & 1.2793 & 2.1819 & 0.2276 & \\
\hline & & scale & 8.5199 & 5.1686 & 14.0440 & 2.1726 & & 8.5199 & 5.1686 & 14.0440 & 2.1726 & \\
\hline & \multirow[t]{3}{*}{ Nonmixture cure model (log-normal) } & theta & 0.1746 & 0.0759 & 0.3527 & NA & \multirow[t]{3}{*}{401.08} & 0.1746 & 0.0759 & 0.3527 & NA & \multirow[t]{3}{*}{401.08} \\
\hline & & meanlog & 2.1736 & 1.5952 & 2.7519 & 0.2951 & & 2.1736 & 1.5952 & 2.7519 & 0.2951 & \\
\hline & & SDlog & 1.0317 & 0.7738 & 1.3756 & 0.1514 & & 1.0317 & 0.7738 & 1.3756 & 0.1514 & \\
\hline & \multirow[t]{3}{*}{ Nonmixture cure model (Gompertz) } & theta & 0.1783 & 0.0884 & 0.3268 & NA & \multirow[t]{3}{*}{414.24} & 0.1783 & 0.0884 & 0.3268 & NA & \multirow[t]{3}{*}{414.24} \\
\hline & & shape & 0.0417 & -0.0195 & 0.1028 & 0.0312 & & 0.0417 & -0.0195 & 0.1028 & 0.0312 & \\
\hline & & rate & 0.0564 & 0.0333 & 0.0952 & 0.0151 & & 0.0564 & 0.0333 & 0.0952 & 0.0151 & \\
\hline \multirow{37}{*}{$\begin{array}{l}\text { Maintenance } \\
\text { olaparib }\end{array}$} & \multirow[t]{2}{*}{ Weibull } & shape & 1.0856 & 0.8786 & 1.3413 & 0.1172 & \multirow[t]{2}{*}{253.52} & 1.5373 & 1.1675 & 2.0242 & 0.2158 & \multirow[t]{2}{*}{256.86} \\
\hline & & scale & 6.7873 & 5.1476 & 8.9493 & 0.9576 & & 24.4556 & 19.2737 & 31.0308 & 2.9712 & \\
\hline & Gamma & shape & 1.3729 & 0.9567 & 1.9701 & 0.2530 & 251.34 & 2.1173 & 1.3708 & 3.2701 & 0.4696 & 254.71 \\
\hline & & rate & 0.2157 & 0.1326 & 0.3510 & 0.0536 & & 0.0960 & 0.0535 & 0.1720 & 0.0286 & \\
\hline & Exponential & rate & 0.1484 & 0.1101 & 0.2001 & 0.0226 & 252.07 & 0.0354 & 0.0248 & 0.0506 & 0.0065 & 262.44 \\
\hline & Log-logistic & shape & 1.9399 & 1.5154 & 2.4833 & 0.2444 & 232.39 & 2.0892 & 1.5679 & 2.7837 & 0.3059 & 252.59 \\
\hline & & scale & 4.0412 & 3.1815 & 5.1332 & 0.4932 & & 17.5577 & 13.6596 & 22.5682 & 2.2490 & \\
\hline & Log-normal & meanlog & 1.4487 & 1.1979 & 1.6995 & 0.1280 & 237.93 & 2.8832 & 2.6265 & 3.1399 & 0.1310 & 251.28 \\
\hline & & SDlog & 0.9159 & 0.7389 & 1.1354 & 0.1004 & & 0.8169 & 0.6293 & 1.0604 & 0.1087 & \\
\hline & Gompertz & shape & -0.0514 & -0.1165 & 0.0138 & 0.0332 & 251.16 & 0.0288 & -0.0084 & 0.0659 & 0.0189 & 262.32 \\
\hline & & rate & 0.1869 & 0.1266 & 0.2759 & 0.0371 & & 0.0257 & 0.0144 & 0.0460 & 0.0076 & \\
\hline & Royston/Parmar spline model & gamma0 & -2.0793 & -2.6344 & -1.5243 & 0.2832 & 253.52 & -4.9147 & -6.2391 & -3.5903 & 0.6757 & 256.86 \\
\hline & (U Knot) & gamma1 & 1.0858 & 0.8561 & 1.3155 & 0.1172 & & 1.5374 & 1.1143 & 1.9604 & 0.2158 & \\
\hline & Royston/Parmar spline model & gamma0 & -3.3244 & -4.3063 & -2.3424 & 0.5010 & 234.45 & -8.3665 & -11.9649 & -4.7680 & 1.8360 & 232.26 \\
\hline & & gamma1 & 2.8794 & 1.9142 & 3.8445 & 0.4925 & & 3.3842 & 1.6327 & 5.1356 & 0.8936 & \\
\hline & & gamma2 & 0.3150 & 0.1700 & 0.4601 & 0.0740 & & 0.5453 & 0.0845 & 1.0061 & 0.2351 & \\
\hline & Royston/Parmar spline model & gamma0 & -3.2913 & -4.3279 & -2.2548 & 0.5289 & 235.96 & -9.0772 & -14.4905 & -3.6639 & 2.7619 & 254.27 \\
\hline & & gamma1 & 2.7541 & 0.8662 & 4.6421 & 0.9633 & & 3.8360 & 0.7951 & 6.8769 & 1.5515 & \\
\hline & & gamma2 & -0.0291 & -1.0954 & 1.0373 & 0.5441 & & 0.8583 & -1.6571 & 3.3736 & 1.2834 & \\
\hline & & gamma3 & 0.3327 & -0.4419 & 1.1072 & 0.3952 & & -0.2791 & -2.7751 & 2.2169 & 1.2735 & \\
\hline & Mixture cure model (Weibull) & theta & 0.1063 & 0.0398 & 0.2544 & NA & 239.11 & 0.1063 & 0.0398 & 0.2544 & NA & 239.11 \\
\hline & & shape & 1.5208 & 1.2295 & 1.8809 & 0.1649 & & 1.5208 & 1.2295 & 1.8809 & 0.1649 & \\
\hline & & scale & 4.8227 & 3.8940 & 5.9729 & 0.5263 & & 4.8227 & 3.8940 & 5.9729 & 0.5263 & \\
\hline & Mixture cure model (gamma) & theta & 0.1086 & 0.0411 & 0.2573 & NA & 235.47 & 0.1086 & 0.0411 & 0.2573 & NA & 235.47 \\
\hline & & shape & 2.3418 & 1.5856 & 3.4586 & 0.4659 & & 2.3418 & 1.5856 & 3.4586 & 0.4659 & \\
\hline & & rate & 0.5480 & 0.3408 & 0.8812 & 0.1328 & & 0.5480 & 0.3408 & 0.8812 & 0.1328 & \\
\hline & Mixture cure model (Exp) & theta & 0.0890 & 0.0263 & 0.2614 & NA & 248.22 & 0.0890 & 0.0263 & 0.2614 & NA & 248.22 \\
\hline & & rate & 0.2037 & 0.1442 & 0.2878 & 0.0359 & & 0.2037 & 0.1442 & 0.2878 & 0.0359 & \\
\hline & Mixture cure model (Log-logistic) & theta & 0.0962 & 0.0306 & 0.2646 & NA & 233.43 & 0.0962 & 0.0306 & 0.2646 & NA & 233.43 \\
\hline & & shape & 2.3880 & 1.8267 & 3.1216 & 0.3264 & & 2.3880 & 1.8267 & 3.1216 & 0.3264 & \\
\hline & & scale & 3.5246 & 2.8298 & 4.3900 & 0.3948 & & 3.5246 & 2.8298 & 4.3900 & 0.3948 & \\
\hline & Mixture cure model (Log-normal) & theta & 0.1023 & 0.0353 & 0.2620 & NA & 232.69 & 0.1023 & 0.0353 & 0.2620 & NA & 232.69 \\
\hline & & meanlog & 1.2396 & 1.0180 & 1.4612 & 0.1131 & & 1.2396 & 1.0180 & 1.4612 & 0.1131 & \\
\hline & & sdlog & 0.7169 & 0.5688 & 0.9035 & 0.0846 & & 0.7169 & 0.5688 & 0.9035 & 0.0846 & \\
\hline & Mixture cure model (Gompertz) & theta & 0.1011 & 0.0360 & 0.2533 & NA & 247.62 & 0.1011 & 0.0360 & 0.2533 & NA & 247.62 \\
\hline & & shape & 0.0753 & -0.0053 & 0.1558 & 0.0411 & & 0.0753 & -0.0053 & 0.1558 & 0.0411 & \\
\hline & & rate & 0.1654 & 0.1075 & 0.2545 & 0.0364 & & 0.1654 & 0.1075 & 0.2545 & 0.0364 & \\
\hline
\end{tabular}

(continued on next page) 


\begin{tabular}{|c|c|c|c|c|c|c|c|c|c|c|c|c|}
\hline Strategies & Distribution & Parameter & Est & L95\% & U95\% & SE & AIC & Est & L95\% & U95\% & SE & AIC \\
\hline \multirow{13}{*}{$\begin{array}{l}\text { Maintenance } \\
\text { olaparib (cont.) }\end{array}$} & \multirow[t]{3}{*}{ Nonmixture cure model (Weibull) } & theta & 0.0925 & 0.0331 & 0.2331 & NA & \multirow[t]{3}{*}{235.84} & 0.0925 & 0.0331 & 0.2331 & NA & \multirow[t]{3}{*}{235.84} \\
\hline & & shape & 1.7922 & 1.4463 & 2.2206 & 0.1960 & & 1.7922 & 1.4463 & 2.2206 & 0.1960 & \\
\hline & & scale & 7.3573 & 5.5111 & 9.8219 & 1.0846 & & 7.3573 & 5.5111 & 9.8219 & 1.0846 & \\
\hline & \multirow{2}{*}{ Nonmixture cure model (Gamma) } & shape & 2.5088 & 1.7480 & 3.6007 & 0.4625 & \multirow{2}{*}{233.65} & 2.5088 & 1.7480 & 3.6007 & 0.4625 & \multirow{2}{*}{233.65} \\
\hline & & rate & 0.3858 & 0.2090 & 0.7121 & 0.1206 & & 0.3858 & 0.2090 & 0.7121 & 0.1206 & \\
\hline & \multirow[t]{2}{*}{ Nonmixture cure model (Exp) } & theta & 0.0263 & 0.0005 & 0.5873 & NA & \multirow[t]{2}{*}{251.16} & 0.0263 & 0.0005 & 0.5873 & NA & \multirow[t]{2}{*}{251.16} \\
\hline & & rate & 0.0514 & 0.0145 & 0.1825 & 0.0332 & & 0.0514 & 0.0145 & 0.1825 & 0.0332 & \\
\hline & Nonmixture cure model (Log-logistic) & theta & 0.0960 & 0.0319 & 0.2550 & NA & 233.25 & 0.0960 & 0.0319 & 0.2550 & NA & 233.25 \\
\hline & \multirow{2}{*}{ Nonmixture cure model (Log-normal) } & meanlog & 1.8009 & 1.3050 & 2.2969 & 0.2531 & \multirow{2}{*}{233.01} & 1.8009 & 1.3050 & 2.2969 & 0.2531 & \multirow{2}{*}{233.01} \\
\hline & & sdlog & 0.8309 & 0.6184 & 1.1164 & 0.1252 & & 0.8309 & 0.6184 & 1.1164 & 0.1252 & \\
\hline & \multirow[t]{3}{*}{ Nonmixture cure model (Gompertz) } & theta & 0.0840 & 0.0273 & 0.2306 & NA & \multirow[t]{3}{*}{247.07} & 0.0840 & 0.0273 & 0.2306 & NA & \multirow[t]{3}{*}{247.07} \\
\hline & & shape & 0.1487 & 0.0623 & 0.2352 & 0.0441 & & 0.1487 & 0.0623 & 0.2352 & 0.0441 & \\
\hline & & rate & 0.0557 & 0.0296 & 0.1048 & 0.0180 & & 0.0557 & 0.0296 & 0.1048 & 0.0180 & \\
\hline
\end{tabular}

Abbreviations: AIC, Akaike information criterion; Est, point estimation; L95\%, lower boundary of 95\% confidence interval; NA, not available; OS, overall survival; PFS, progression-free survival; U95\%, upper boundary of $95 \%$ confidence interval. 